\title{
Genomics shifts focus to rare diseases
}

\section{COLD SPRING HARBOR, NEW YORK}

Genome sequencing may finally be living up to its promise of pinpointing genetic mutations that bear on treatment for individual patients. But the breakthroughs are not coming from the DNA analysis of common diseases with complex genetic origins, which has been the obsession of genomics for nearly the past decade. Instead, many genome scientists are turning back to study rare disorders that are traceable to defects in single genes, and whose causes have remained a mystery.

The change is partly a result of frustration with the disappointing results of genomewide association studies (GWAS). Rather than sequencing whole genomes, GWAS studies examine a subset of DNA variants in thousands of unrelated people with common diseases. Now, however, sequencing costs are dropping, and whole genome sequences can quickly provide in-depth information about individuals, enabling scientists to locate genetic mutations that underlie rare diseases by sequencing a handful of people.

"Years ago, people were using families and mapping approaches to distil down to a region where they thought a causative gene was," says Elaine Mardis, a director of the Genome Sequencing Center at Washington University in St Louis, Missouri. "Fast-forward 12 years, and you've got sort of the same thing going on, except with new technology that gives us much higher resolution and speed."

The change was showcased at the second Personal Genomes meeting, held in Cold Spring Harbor, New York, last week. At the same meeting last year, most speakers focused on the genome of scientist James Watson - one of only four fully sequenced individuals available at that time (see Nature 455, 1014; 2008). Now, about 50 individual genomes are published or in production, many of mostly anonymous patients with medical needs, estimates

"I now think that we're going to get there by understanding a whole lot of these Mendelian diseases." Richard Gibbs, director of the Human Genome Sequencing Center at Baylor College of Medicine in Houston, Texas.

The first genomes of patients came in cancer, but scientists are now quickly moving into less high-profile diseases. For instance, together with Baylor's James Lupski, Gibbs reported that they had sequenced the genomes of patients in one family with a familial neuropathy - a disorder marked by muscle weakness and pain - and found defects in a single gene that could account

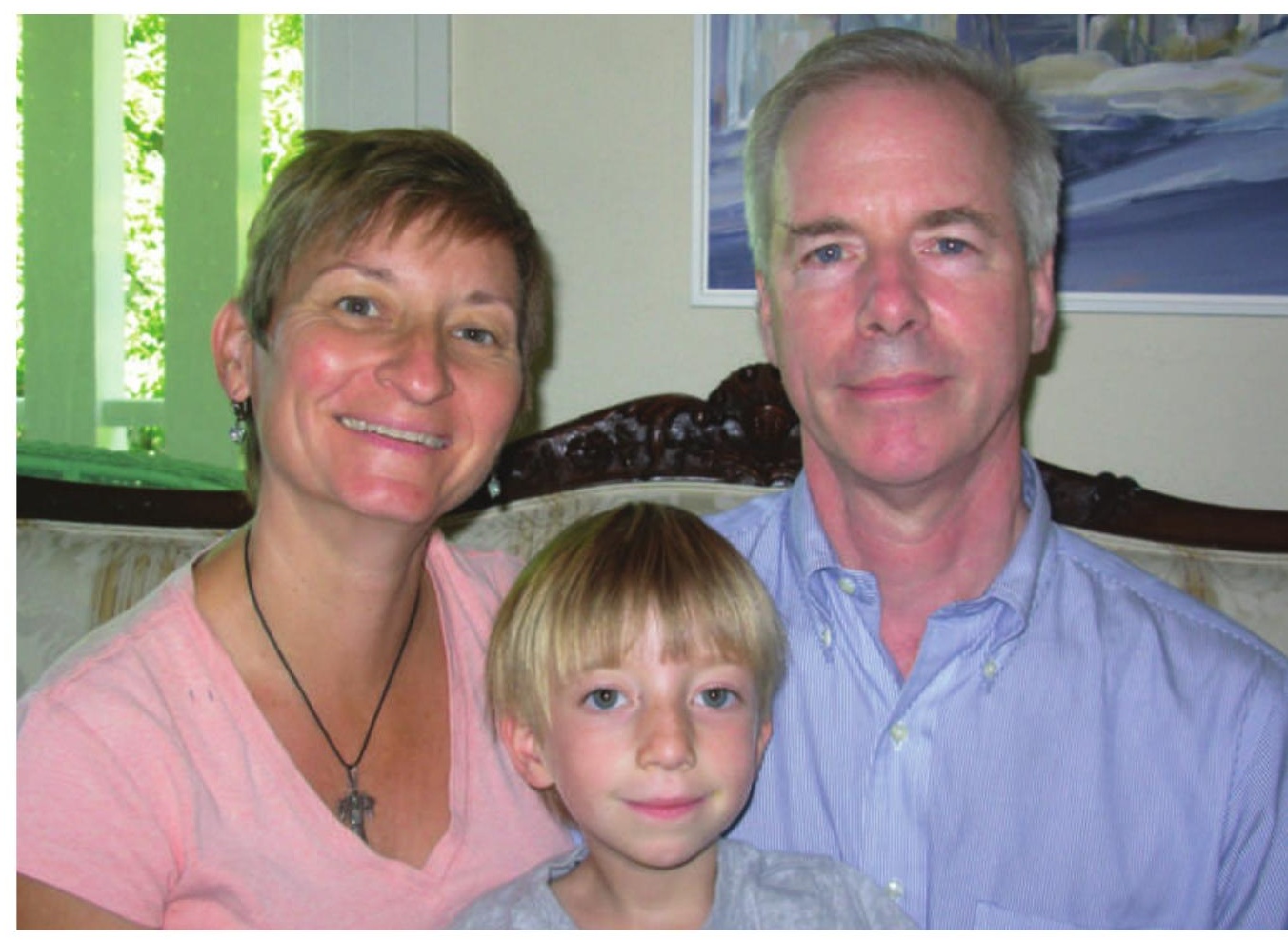

Hugh Rienhoff sequenced family transcriptomes to try to diagnose his daughter Bea's genetic disease.

for the family's condition. Matthew Bainbridge, a student in Gibbs's lab, reported that he had found a genetic glitch that is probably responsible for an inherited form of ataxia, a disorder affecting bodily coordination, by sequencing the exome, or set of all protein-coding genes, of two distant relatives with the disease. And Jay Shendure of the University of Washington in Seattle used a separate exome-sequencing strategy to find the gene that could be responsible for Miller syndrome, marked by head and facial abnormalities.

Meanwhile, Richard Lifton of Yale University in New Haven, Connecticut, reported a striking example of how genome sequencing can help patients. A doctor asked Lifton to study a sickly infant who appeared to have a kidney disease. But Lifton's group sequenced the exomes of the infant and some family members and found a genetic variant in a gene, called SLC26A3, that causes congenital chloride diarrhoea, a treatable disease. Lifton informed the doctor, who reported that, indeed, the infant had had hourly bouts of diarrhoea.

The meeting also heard from bioentrepre- neur Hugh Rienhoff, a California father who has sequenced his daughter's transcriptome - the readout of protein templates expressed in her cells - as well as his wife's and his own to find the cause of her undiagnosed genetic disease (see http://tinyurl.com/mf6oxs). The effort has yielded a list of mutated genes that could cause her unique collection of symptoms, including long hands and feet and a cleft uvula, says Rienhoff, who trained as a clinical geneticist.

He argues that studies like his are promising first steps to understanding more complex diseases. "First, let's figure out the diseases that are $100 \%$ genetic and then go after the diseases that aren't $100 \%$ genetic," he says, pointing out that there are about 3,000 Mendelian diseases - inherited disorders caused by defects in single genes - of which the genetic causes are not known. "It's the new new thing, which is old: studying the rare stuff because it bears on the common stuff," he says.

"The issue is how we're going to understand the architecture of common disorders," agrees Gibbs. "Three or four years ago I thought it was going to be because of GWAS studies, but I now think that we're going to get there by understanding a whole lot of these Mendelian diseases." 
Leslie Biesecker, who heads the ClinSeq study at the National Human Genome Research Institute in Bethesda, Maryland, is uncovering evidence to support that idea. ClinSeq has enrolled 725 patients and sequenced 251 of their genes, including two genes that, when mutated, cause familial hypercholesterolaemia (FH), a disease marked by high cholesterol levels. In seven patients with high cholesterol, Biesecker found mutations in the two FH genes, even though some of the patients had not been diagnosed with the condition. Biesecker estimates that 75-100 family members of these patients probably have undiagnosed $\mathrm{FH}$, and is trying to contact them.

"If we can leverage the finding to diagnose and treat 75-100 people for $\mathrm{FH}$, I can go to bed at night feeling like our sequencing money has been well spent," he says.

\section{Plummeting prices}

Cost is still a roadblock to broader sequencing, however, so scientists at the meeting were interested in a report from collaborators of Complete Genomics, of Mountain View, California, which says it will dramatically cut the price of sequencing by offering human-genome sequences for US $\$ 5,000$ next year. The company sequenced a family of four people for $\$ 20,000$ apiece, including two children with undiagnosed symptoms, for the Institute for Systems Biology in Seattle, Washington, whose founder, Lee Hood, is on Complete Genomics' scientific advisory board. Lee Rowen, a researcher at the institute, said that its analysis of Complete Genomics' data has yielded three candidate genes for the children's disorders. "We were happy with what they gave us," Rowen says.

However, she also estimates that there could be roughly 10-20 errors per megabase of DNA, which would translate to tens of thousands of errors per genome. The group is resequencing each error to try to pinpoint the error rate more closely.

That left many in the audience unsatisfied. "What use is a $\$ 20,000$ genome if you have to spend even more money to figure out if it's right or not?" Mardis said.

Some contenders in the crowded sequencing field hope to ultimately push costs as low as hundreds of dollars per genome. Until then, the ultimate payoff on the promise of genomics - a true understanding of common diseases - remains over the horizon. But for now, a down payment appears to have been made.

Erika Check Hayden

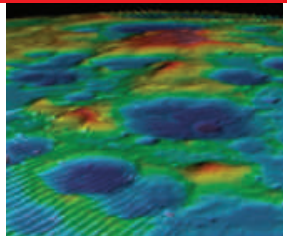

WATER ON THE MOON?

Lunar missions find evidence of ice and hydrated minerals. www.nature.com/news

\section{The elephant and the neutrino}

India's environment minister Jairam Ramesh will visit the site of a proposed underground neutrino laboratory next month, to try to break the impasse between physicists and environmentalists over its construction.

The US\$160-million India-based Neutrino Observatory (INO) was to have been completed by 2012 to study the elusive particles known as neutrinos (see Nature 450,13 ; 2007). But its construction is mired in controversy over the wisdom of locating the facility in prime elephant and tiger habitat at Singara in the Nilgiri Biosphere Reserve, 250 kilometres south of Bangalore.

The observatory applied for permission to begin construction at the Singara site in 2006; "there has been no reply to date," says project spokesman Naba Mondal, a physicist at the Tata Institute of Fundamental Research in Mumbai. "All I know is we have not cleared it," says A.S. Balanathan, principal chief conservator of forest for the state of Tamil Nadu, who declined to comment further.

Last month, 11 leading physicists, including Nobel laureates Sheldon Glashow and Masatoshi Koshiba, wrote to India's prime minister Manmohan Singh urging that the project move forward. "The INO will bring more big science to India and enhance India's role as an important player in frontline science," they wrote. Meanwhile, prominent Indian conservationists are circulating and signing a letter laying out their concerns and asking that the observatory be sited elsewhere.

The Nilgiri reserve includes more than 5,500 square kilometres of continuous forest cover and six protected areas. The proposed location for the INO comes as close as 7 kilometres to the edge of one of the sanctuaries. The project involves digging out a 120-metre-long cavern at the end of a 2-kilometre-long tunnel inside a mountain. The cavern will house a magnetized iron calorimeter to detect the muons that are produced occasionally when neutrinos interact with matter.

The controversy stems from disagreements over the impact of the tunnelling and the increased human population on the fragile ecosystem. "Transporting the estimated 630,000 tonnes of debris and 147,000 tonnes of construction material would require about 156,000 truck trips through 35 kilometres of forest - and two tiger reserves," says the NBR Alliance, a group of Indian organizations concerned about the reserve. This means 468,000 hours of disturbance to animal movement routes, the alliance estimates.

The INO team "could hardly have picked a site in India more likely to damage wildlife," says John Seed, an Australian environmentalist who has researched elephant habitats in India. "As well as being home to the largest single population of Asian elephants in the world," he says, "the Nilgiri is also one of the most important tiger habitats in the country."

Mondal disputes the tally of construction debris and says that the project will limit the number of daily truck trips and restrict them to daytime. But Priya Davidar, an ecologist at Pondicherry University, says that the environmental impact assessment the project submitted to state officials is seriously flawed. Davidar is president of the Association for Tropical Biology and Conservation, based in Washington DC, which passed its own resolution urging the Indian government

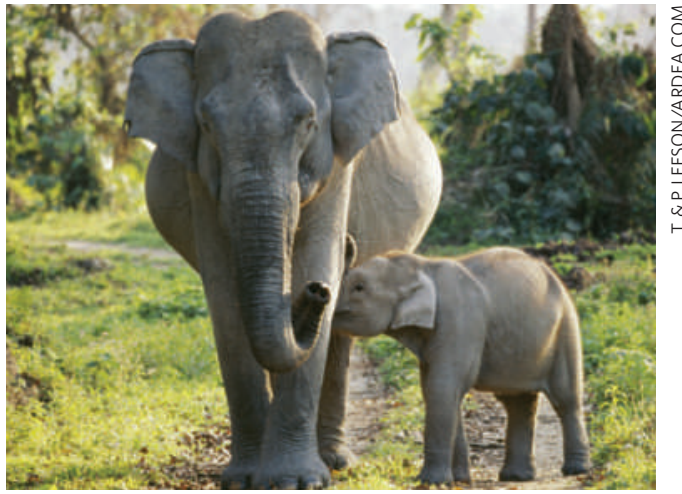

Big hurdles for India's neutrino-detection lab.

not to permit construction and to look for an alternative site.

Davidar criticizes the project for limiting its search to only two sites; a better location, she says, would have been the Kolar gold mine in neighbouring Karnataka state, used for neutrino detection in 1965. But the Kolar mine is now closed and filled with water, and is not suitable for lowering heavy materials down, says Mondal. He says that after considering other sites, his team, along with the Geological Survey of India, identified Singara as "the best available site for locating the INO, based on safety, seismicity, as well as year-round accessibility".

The minister will visit on 10 October. If a construction permit is denied, INO may have to start looking for another site. Killugudi Jayaraman 